\title{
CRT auch bei leichter Herzinsuffizienz?
}

\begin{abstract}
Die aktuellen Leitlinien der kardiologischen Fachgesellschaften empfehlen die Implantation eines Defibrillators (ICD) mit kardialer Resynchronisationstherapie (CRT) bei Patienten mit Herzinsuffizienz in den Stadien NYHA III-IV, einer eingeschränkten linksventrikulären Ejektionsfraktion (LV-EF) $\leq 35 \%$ und einer Verbreiterung des QRS-Komplexes $\geq 120 \mathrm{~ms}$ bei Vorliegen von Sinusrhythmus. Die MADIT-Studie untersuchte, ob die CRT auch Patienten mit nur milden Herzinsuffizienzsymptomen (NYHA I-II) nützt.
\end{abstract}

In der prospektiven MADIT-Studie wurde untersucht, ob die primärprophylaktische Implantation eines ICD-CRT die Sterblichkeit und das Auftreten nicht tödlicher Herzinsuffizienz-Ereignisse bei Patienten mit Herzinsuffizienz in den Stadien NYHA I-II, eingeschränkter LV-EF <30\% und ORS-Verbreiterung $>130 \mathrm{~ms}$ im Vergleich zur Implantation eines einfachen ICD ohne CRT verringert.

1820 Patienten ( $n=1089$ in der ICD-CRTGruppe) mit ischämischer (ca. 55\%) oder nicht ischämischer Kardiomyopathie (ca. 45\%) wurden in die Studie eingeschlossen. Die mittlere LV-EF betrug in beiden Gruppen $24 \%$. Rund $70 \%$ der Patienten hatten einen kompletten Linksschenkelblock. Zwei Drittel der Patienten wiesen eine QRS-Dauer $\geq 150 \mathrm{~ms}$ auf. Es fand sich kein Unterschied hinsichtlich der konservativen medikamentösen Behandlung der Herzinsuffizienz (93\% Betablocker, 97\% ACEHemmer oder Angiotensin-Rezeptor-Antagonist).

Die Studie wurde nach einem mittleren Nachbeobachtungszeitraum von 2,4 Jahren terminiert, nachdem die prädefinierten Effektivitätskriterien erfüllt waren. In diesem Zeitraum wurde der primäre Endpunkt (Tod jeglicher Ursache und nicht tödliche Herzinsuffizienz-Ereignisse) von $17,2 \%$ der Patienten aus der ICD-CRT-Gruppe und 25,3\% der Patienten aus der ICD-Gruppe erreicht. Das entspricht einer relativen Risikoreduktion von 34\% ( $p=0,001)$.

Dieser Vorteil durch die ICD-CRT-Therapie resultierte aus einem verminderten

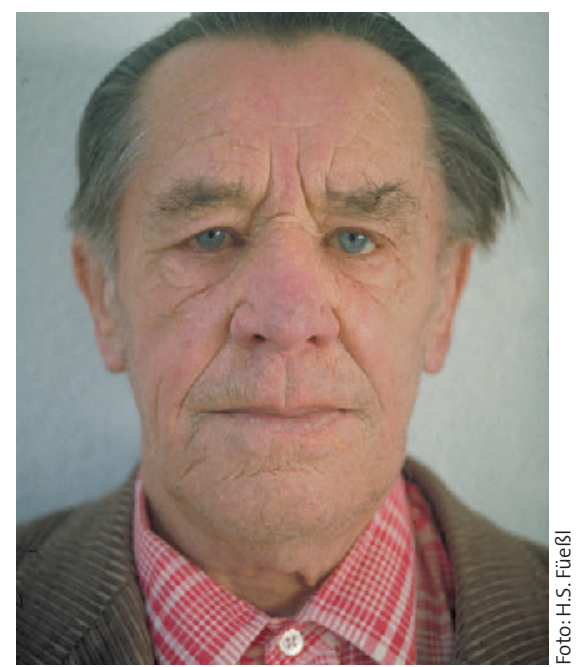

Akrale Zyanose bei einem Patienten mit Herzinsuffizienz.

Auftreten von Herzinsuffizienz-Exazerbationen (13,9 vs. 22,8\%; HR=0,59; $p \leq 0,001$ ), wohingegen die Mortalitätsrate nicht signifikant unterschiedlich war (7,3 vs. 6,8\%; $\mathrm{HR}=1 ; \mathrm{p}=0,99)$. Dies galt sowohl für $\mathrm{Pa}$ tienten mit ischämischer Kardiomyopathie als auch für jene mit nicht ischämischer Genese der Herzinsuffizienz.

In einer Subgruppenanalyse wurde festgestellt, dass insbesondere Patienten mit einer QRS-Dauer $\geq 150 \mathrm{~ms}$ von der CRT profitierten $(H R=0,48)$ und Frauen unabhängig von der QRS-Dauer ( $R R=0,37)$.

$\mathrm{Zu}$ beachten ist, dass perioperative Komplikationen (Hämatom, Infektion, Pneumothorax, Perikarderguss oder Repositionierung der LV-Sonde) in der ICDCRT-Gruppe deutlich häufiger auftraten (10,6 vs. $4 \%$ ).

II Kommentar: Die Ergebnisse der Studie sind sehr bemerkenswert, da sie den präventiven Nutzen der CRT bei Patienten mit milder Herzinsuffizienz ischämischer und oder nicht ischämischer Genese erneut belegte.

Einschränkend muss allerdings angemerkt werden, dass immerhin rund 10\% der Patienten in der Vergangenheit im NYHA-Stadium III gewesen waren, bevor sie in die Studie eingeschlossen wurden.
Dieser Teil des Studienkollektivs ähnelt somit eher den klassischen CRT-Patienten.

Die echokardiografischen Resultate nach zwölf Monaten zeigten eine Verbesserung der LV-EF um 11\% durch die CRT (gegenüber 3\% in der ICD-Gruppe) im Sinne eines „reverse remodeling“. Die Überlegenheit der CRT in diesem Patientenkollektiv resultierte daher nicht aus einer etwaigen Exazerbation in der ICD-Gruppe (z.B. durch die rechtsventrikuläre Stimulation), sondern aus einer echten Verbesserung der kardialen Pumpfunktion durch die CRT. Auch wenn sich kein Unterschied hinsichtlich der jährlichen Mortalitätsrate von ca. $3 \%$ fand, wurde eine Vielzahl von Hospitalisierungen wegen Herzinsuffizienz vermieden. Insgesamt mussten nämlich $87 \%$ aller Patienten mit einer Verschlechterung der Herzinsuffizienz hospitalisiert werden, und die relative Risikoreduktion durch die CRT betrug $41 \%$.

Dieses Patientenkollektiv ist in den aktuellen Leitlinien zur CRT bisher nicht repräsentiert. Es bleibt abzuwarten, ob die Fachgesellschaften eine präventive CRT bei Patienten mit milder Herzinsuffizienz jedweder Genese, LV-EF $\leq 30 \%$ und ORS $\geq 130$ ms empfehlen werden.

In Zeiten schwindender Ressourcen im Gesundheitssystem sollte jede potenzielle Innovation einer ökonomischen KostenNutzen-Evaluation unterzogen werden und medizinische Alternativen zur Prävention der Herzinsuffizienz-Exazerbation (z.B. Home-Monitoring) geprüft werden. Aus den vorliegenden Daten erschient es zunächst sinnvoll, die CRT bei Herzinsuffizienz in den Stadien NYHA I-II nur Patienten mit einem besonders breiten QRS-Komplex $\geq 150 \mathrm{~ms}$ und insbesondere Frauen zu empfehlen.

Dr. med. Boris Schmidt, Prof. Dr. med. Karl-Heinz Kuck, Asklepios Klinik St. Georg, Hamburg II

II Moss AJ et al. Cardiac-Resynchronization Therapy for the Prevention of Heart-Failure Events. N Engl J Med 2009;361:1329-1338 